\title{
LA TEORÍA DE LA SELECCIÓN NATURAL \\ DARWINIANA Y LA GENÉTICA \\ DE POBLACIONES*
}

\section{THE THEORY OF NATURAL SELECTION AND POPULATION GENETICS}

\author{
Santiago GinNOBILI** \\ IEC - Instituto de Estudios sobre la Ciencia y la Tecnología \\ Universidad Nacional de Quilmas. Argentina
}

\begin{abstract}
Resumen: Para algunos la selección natural se identifica con las diferencias de éxito de distintos organismos en la reproducción diferencial. Si esto fuese así, el principio de Hardy-Weinberg, por permitir determinar con bastante precisión bajo ciertos supuestos que la frecuencia génica en una población no es la esperada, podría ser visto como una versión cuantitativa de la selección natural cualitativa propuesta por Darwin. Es mi intención mostrar, a través del análisis de explicaciones dadas por Darwin, que la selección natural es más que la mera diferencia en el éxito en la reproducción diferencial e investigar algunas relaciones entre la teoría de la selección natural y la genética de poblaciones.
\end{abstract}

Palabras ClaVE: Genética de poblaciones, principio de Hardy-Weinberg, selección natural, teoría de la evolución.

ABSTRACT: Natural selection is often identified with success differences in differential reproduction. If this were the

* Agradezco los comentarios de Pablo Lorenzano y Daniel Blanco a versiones previas de este trabajo. Este trabajo fue realizado con la ayuda del proyecto de investigación PICT Redes 2006 No 2007 de la Agencia Nacional de Promoción Científica y Tecnológica.

** UBA - UNQ - CONICET. Para contactar al autor escriba a santi75@gmail.com. 
case, Hardy-Weinberg's principle could be regarded as a quantitative version of Darwin's qualitative Natural Selection Theory, since it allows the determination of the expected genetic frequency of a population, under certain assumptions. My aim here is to show that Natural Selection is more than the mere difference in the differential reproduction success through the analysis of the explanations given by Darwin, and to explore the relations between the theory of Natural Selection and Populations Genetics.

KEYWORDS: Hardy-Weinberg principle, natural selection, population genetics, theory of evolution.

\section{Introducción}

Para algunos la teoría de la selección natural (TSN en adelante) se define únicamente a partir de diferencias en el éxito de distintos organismos en la reproducción diferencial. Si esto fuese así, el principio de Hardy-Weinberg (HW en adelante), por permitir determinar (como veremos) con bastante precisión, bajo ciertos supuestos, que la frecuencia génica en una población no es la esperada, permitiría cuantificar la acción de la selección natural. Incluso, podría considerarse que la genética de poblaciones (GP en adelante) brinda una versión cuantitativa de la teoría de la selección natural cualitativa propuesta por Darwin ${ }^{1}$.

Así, por ejemplo, en su conocido libro de texto sobre evolución, Futuyma afirma: «Si los alelos difieren en sus tasas de reemplazo, sus frecuencias pueden cambiar. Este fenómeno es llamado "selección”" (Futuyma 1986, pp. 86-87). Por otro lado, y en una disciplina diferente, el reconocido historiador de la ciencia Gayon dice: «[Con el descubrimiento de Hardy y Weinberg] la selección natural no es más un principio fundamental, sino un parámetro que mide una de las tantas fuerzas de cambio» (Gayon 1998, p. 321).

1 La utilización del término "teoría» en este artículo se referirá en todos los casos a lo que en la metateoría estructuralista es llamado «red teórica» (Balzer, Moulines, y Sneed 1987, cap. IV). Presupondré en este artículo que TSN y GP son redes teóricas. Argumentaré, por supuesto, que se trata de redes teóricas distintas. 
Ésta idea abonaría el papel central que para algunos tiene la HW en la teoría de la evolución en general. Ésta sería, según ciertos autores, la ley fundamental, no sólo de la genética de poblaciones, sino además de la teoría evolutiva ${ }^{2}$. Este es el caso de Michael Ruse quien otorga a GP capacidad de unificar a toda la biología evolutiva (Ruse 1973, cap. 4) y parece estar presupuesto, también, en autores que pretenden haber reconstruido la teoría evolutiva reconstruyendo sólo GP (p.e. Barbadilla 1990; Lloyd 1994; Thompson 1989).

Mi objetivo en este artículo es defender que GP y TSN son dos teorías distintas aunque vinculadas, alguno de cuyos vínculos pretendo mostrar. Un colorario del análisis que sigue consistirá en que GP no puede ser la teoría fundamental de la teoría de la evolución, pues existe al menos una teoría, TSN, que no puede ser incluida bajo GP.

En I mostraré informalmente una reconstrucción de TSN. Aunque esta reconstrucción se encuentra inacabada y es informal, basta para sostener el punto defendido. En II presentaré esquemáticamente HW en su formulación habitual. La presentación de TSN es más elaborada porque en la comprensión de su estructura radica mi defensa de su independencia de GP. En III, luego de dejar en claro que se trata de teorías distintas, explicaré por qué se ha pensado que era posible aplicar TSN a partir de GP, recurriendo a una analogía con la mecánica clásica. En IV presentaré alguno de los vínculos por los que se relacionan TSN y GP. En $\mathrm{V}$, finalmente, presentaré mis conclusiones.

\section{La teoría de la selección natural}

Comenzaré exponiendo los conceptos fundamentales de la teoría de la selección natural y lo que se pretende explicar con ésta. Esto permitirá, por un lado, establecer cierto vocabulario para poder discutir cuestiones subsiguientes sin ambigüedad y permitirá establecer con claridad la diferencia y los vínculos con GP. Aunque, curiosamente, TSN es mucho más simple de comprender que GP,

2 Si bien yo sólo intentaré mostrar que no puede tratarse de la ley fundamental de la teoría de la evolución, tampoco es adecuado considerar HW como la ley fundamental de GP. Para un tratamiento de esta cuestión ver Lorenzano (2008). Aquí sólo supondré que HW es una ley con un papel central en GP. 
me veré forzado a tratar la cuestión de los conceptos fundamentales de TSN con mayor profundidad y extensión. La razón por la que tales teorías se confunden tiene que ver, bajo mi punto de vista, con una mala comprensión de la estructura de TSN y no con una mala comprensión de GP. Extraeré los conceptos fundamentales de TSN a partir de un caso de aplicación del mismo Darwin ${ }^{3}$.

Cómo es bien sabido, lo que Darwin quiere explicar con TSN es cierta adecuación o ajuste de los organismos al medio ambiente.

Por ejemplo:

La jirafa, con su gran estatura, sus muy largos cuello, patas delanteras, cabeza y lengua, tiene su estructura bellamente adaptada para comer en las ramas más altas de los árboles. Puede por eso obtener comida fuera del alcance de otros ungulados que habitan el mismo lugar; y esto debe ser una gran ventaja durante períodos de escasez (Darwin 1872, p. 161).

La forma en que Darwin explica la fijación de este rasgo en la población de jirafas es la siguiente:

En la naturaleza, en el origen de la jirafa, los individuos que comiesen más alto y que pudiesen durante los periodos de escasez alcanzar aunque sea una pulgada o dos por sobre los otros, serían frecuentemente preservados [...]. El que los individuos de la misma especie muchas veces difieren un poco en la longitud relativa de todas sus partes, puede comprobarse en muchas obras de historia natural en las que se dan medidas cuidadosas. Estas pequeñas diferencias en las proporciones, debidas a las leyes de crecimiento o variación, no tienen la menor importancia ni utilidad en la mayor parte de las especies. Pero en el origen de la jirafa debe hacer sido diferente, considerando sus probables hábitos de vida; pues aquellos individuos que tuviesen alguna parte $o$ varias partes de su cuerpo un poco más alargadas de lo corriente, hubieron en general de sobrevivir. Se habrán cruzado y dejado descendencia que habrán heredado las mismas peculiaridades corpóreas, o la tendencia a variar de nuevo en la misma manera, mientras que los individuos menos favorecidos en los

3 Para un análisis más extenso de la estructura de TSN ver (Ginnobili 2006, 2007a, 2007b, 2009, por aparecer; Ginnobili y Carman 2008). 
mismos aspectos, habrán sido más propensos a perecer (Darwin 1872, p. 161, itálicas mías).

Si consideramos, con los estructuralistas, que la ley fundamental de TSN es aquel enunciado en el que aparecen los conceptos fundamentales de TSN relacionados (Balzer, Moulines, y Sneed, 1987, p. 19; Lorenzano 2005), podemos extraer una instanciación de TSN a partir de la explicación citada:

Las jirafas con cuello, patas delanteras, cabeza y lengua de mayor longitud son más efectivas al alimentarse de las ramas más altas de los árboles, mejorando su supervivencia y mejorando, en consecuencia, su éxito reproductivo diferencial.

Nada de este enunciado parece superfluo. Si quitamos alguna de sus partes, la explicación brindada con él quedaría trunca. Si se quita la función dada al rasgo por el organismo, la de alcanzar las ramas altas de los árboles, no sabríamos por qué tal rasgo podría mejorar la supervivencia. Podría mejorarla por otro motivo, por ejemplo, permitiendo asustar a posibles predadores. Ésta sería una explicación alternativa y competidora de la ofrecida por Darwin. Sí quitamos la mejora en la supervivencia, quedaría indeterminada la relación que hay entre el rasgo y el éxito reproductivo. Como veremos ahora, esta conexión no siempre es a través de una mejora en la supervivencia. En todo caso, alguien, con toda razón, podría objetar que es incompleto. No se hace referencia alguna, por ejemplo, al ambiente. En estas circunstancias no pretendo brindar una reconstrucción completa., si se me concede que al menos estos elementos señalados deben estar contenidos en la ley y que funcionan del modo en el que sostengo que funcionan, ya es suficiente para el punto defendido en este trabajo.

Por abstracción de este enunciado, nos vamos acercando a lo que considero que es la ley fundamental de TSN:

Los individuos con rasgos que cumplen con mayor efectividad cierta función ${ }^{4}$, mejoran su supervivencia mejorando su éxito en la reproducción diferencial.

${ }^{4}$ Para un análisis del papel de los conceptos funcionales en TSN ver Ginnobili (2009). 
La ley fundamental de TSN tendría al menos tres componentes:

- El rasgo que cumple de manera más adecuada una función

- El éxito reproductivo diferencial.

- La conexión entre el rasgo adecuado y el éxito reproductivo, que en este caso se da por una mejora en la supervivencia.

Este tercer componente, que en este caso particular aparece como supervivencia, considero que es el concepto propuesto por la teoría de la selección natural para explicar lo que pretende. Se trata de un concepto abstracto que recibe diferentes interpretaciones y que permite confeccionar a Darwin explicaciones distintas. La conexión entre el rasgo adecuado al ambiente y el éxito en la reproducción diferencial no siempre es a través de una mejora en la supervivencia. Por ejemplo:

Generalmente, los machos más vigorosos, los que están mejor adecuados a su situación en la naturaleza dejarán más descendencia, pero en muchos casos la victoria depende no tanto del vigor natural como de la posesión de armas especiales limitadas al sexo masculino. Un ciervo sin cuernos, un gallo sin espolones, habrían de tener pocas probabilidades de dejar numerosa descendencia. La selección sexual, dejando siempre criar al vencedor, pudo, seguramente, dar valor indomable, longitud a los espolones, fuerza al ala para empujar la pata armada de espolón [...] [Darwin (1859), p. 88].

El enunciado legaliforme presupuesto en este caso sería:

Los gallos de espolones más efectivos para luchar con gallos del mismo sexo tienden a emparejarse más, mejorando, en consecuencia, su éxito en la reproducción diferencial 5 .

En otros casos la explicación puede no acudir ni a mejoras en la supervivencia ni a mejoras en la capacidad de atraer parejas. Por ejemplo:

5 Muchos han discutido las relaciones entre selección natural y selección sexual. Es posible mostrar con facilidad la estructura común entre las explicaciones que utilizan ambos mecanismos y, por lo tanto, existe un sentido interesante en que se puede afirmar en que son casos de aplicación de una y la misma teoría. Para discusiones más profundas de esta cuestión se puede consul$\operatorname{tar}$ (Endler 1986; Ginnobili 2006, por aparecer). 
Las plantas que produjesen flores con las glándulas y nectarios mayores y que segregasen más néctar serían las visitadas con mayor frecuencia por insectos y las más frecuentemente cruzadas, y de este modo, a la larga, adquirirían ventaja y formarían una variedad local [Darwin, 1859, p. 92].

El enunciado legaliforme supuesto sería:

Las plantas que producen flores más atractivas a los insectos tienden a mejorar su fecundidad mejorando, en consecuencia, su éxito en la reproducción diferencial.

Si llamamos al concepto en cuestión «aptitud ${ }^{6}$ la ley fundamental de TSN podría ser:

Los individuos con rasgos que cumplen con mayor efectividad cierta función, mejoran su aptitud, mejorando su éxito en la reproducción diferencial.

No discutiré en esta ocasión el estatus del concepto de aptitud. Basta con señalar que se trata de un concepto abstracto que recibe diferentes interpretaciones en las leyes especiales de TSN, permitiendo realizar explicaciones selectivas diversas. Discutiré, porque es fundamental en este trabajo, el estatus de teoricidad de los otros dos conceptos.

Normalmente los conceptos teóricos de una teoría dada permiten explicar hechos descriptos o interpretados a partir de los conceptos no teóricos de esa teoría. Así, los conceptos de fuerza y masa en la mecánica clásica permiten explicar las aceleraciones de las partículas. Fuerza y masa son conceptos teóricos en la mecánica clásica, puesto que no es posible aplicarlos independientemente de esta teoría, mientras que aceleración es un concepto no teórico en la mecánica clásica, puesto que sí es posible aplicarlo independientemente de esta teoría ${ }^{7}$.

${ }^{6}$ La elección terminológica puede resultar algo extraña al lector. Pero no puedo superar esta dificultad fácilmente. O elijo términos alejados de los normalmente utilizados para hablar de la selección natural volviendo en ese caso al texto más difícil de leer, o elijo términos cercanos, y corro el riesgo de ser mal interpretado o de que la terminología moleste a los que la utilizan de un modo distinto. Esta dificultad surge del hecho, que cualquiera que haya cometido la tarea del análisis o la reconstrucción de la teoría evolutiva conoce, de que en este ámbito hay más conceptos presupuestos que términos para expresarlos (un artículo famoso al respecto es el de Gould y Vrba (Gould y Vrba 1982).

7 Utilizo de manera informal la distinción entre conceptos t-teóricos y t-no teóricos del estructuralismo metateórico (Balzer, Moulines, y Sneed 1987). 
Que un organismo posea un rasgo que permita realizar una función con cierta efectividad debiera poder ser establecido con independencia de TSN, pues para explicar esto es para lo que Darwin propuso esta teoría. Y así es afectivamente, que las jirafas utilizan el cuello para alcanzar las ramas altas de los árboles puede ser determinado por el estudio del comportamiento de las jirafas sin necesidad de la postulación de ningún mecanismo evolutivo y con independencia del origen histórico filogenético del rasgo. La posibilidad de determinar independientemente de TSN estos conceptos también se puede apoyar en el hecho historiográfico de que esto mismo es lo que los teólogos naturales querían explicar apelando a un diseñador inteligente (Blanco 2008).

También puede ser determinado con independencia de TSN el éxito reproductivo de los organismos. Sea como fuere que se realice esta medición, surgirá mediata o inmediatamente del conteo de descendencia fértil o viable de estos organismos sin suponer TSN.

Así, TSN tiene al menos dos conceptos no teóricos: adecuación y éxito reproductivo. Siendo así, con TSN es posible dar dos tipos de explicaciones diferentes:

- Explicación del surgimiento de la adecuación. Para esto fue propuesta TSN por Darwin, para explicar la fijación de adecuaciones al ambiente sin necesidad de postular ningún tipo de diseñador inteligente. Para esto apela a los conceptos de aptitud y de éxito reproductivo. La explicación citada más arriba acerca del cuello de la jirafa sería un caso de este tipo.

- Explicación de las diferencias en el éxito en la reproducción diferencial: Es posible también explicar por qué determinados individuos tienen más éxito que otros apelando a los conceptos de aptitud y de adecuación.

A Darwin no le interesaba particularmente este último tipo de explicación, pues no tenía forma de determinar con claridad el éxito reproductivo de los organismos vivos. Esta situación, como veremos, cambió abruptamente con el tiempo. Con el descubrimiento de HW y con el decaimiento de las explicaciones que incluían diseñadores inteligentes, al menos dentro del seno de la biología, ésta es la función explicativa más importante de TSN. Pues es posible ahora establecer cuantitativamente con cierta precisión la frecuencia esperada de rasgos en una población si se cumplen ciertos supuestos. Si la frecuencia no es la esperada, una 
de las posibles explicaciones es, justamente, que existe una presión selectiva a favor de los organismos que poseen ciertos rasgos. Veremos esto con mayor detenimiento.

\section{Genética de poblaciones}

Mi intención es ahora dar una breve presentación de GP, en particular, de $\mathrm{HW}$, funcional a los fines de este trabajo. En este caso no la reconstruiré, sino que seguiré simplificadamente las presentaciones habituales de los libros de texto de biología evolutiva.

Hardy y Weinberg, de un modo independiente mostraron, a comienzos del siglo XX, cuál debiera ser la frecuencia genética esperada en una población dada que cumple con las siguientes condiciones (Futuyma 1986, p. 83-87):

1. El tamaño de la población es infinito. En una población finita la frecuencia de los alelos puede fluctuar de generación en generación debido a eventos azarosos (deriva génica).

2. Los individuos se aparean unos con otros azarosamente.

3. No ocurren mutaciones.

4. No hay migraciones de ningún tipo.

5. Los alelos son igualmente competentes para hacer copias de sí mismos, entrando en el pool genético de los gametos.

En una población ideal en la que se cumplieran estos supuestos Hardy y Weinberg mostraron matemáticamente para un único gen que posee dos alelos $(A$ y $a)$, que las frecuencias relativas de estos alelos no cambiaran de una generación a otra. El reservorio génico con respecto a estos alelos estará en el estado de equilibrio expresado en la ecuación: $\mathrm{p}^{2}+2 \mathrm{pq}+\mathrm{q}^{2}=1$, en donde $\mathrm{p}^{2}$ permitirá establecer la frecuencia de los genotipos $A A$ - los homocigotos dominantes-, 2pq la frecuencia de los fenotipos $A a$ - los heterocigotos - y q $\mathrm{q}^{2}$ la frecuencia de los genotipos a $a$ - los homocigotos recesivos-.

Si en una población dada la frecuencia génica para un gen con dos alelos no es la esperada, entonces podemos inferir que algunos de los supuestos señalados no está siendo cumplido. Supongamos un caso en el que una población deter- 
minada es lo suficientemente grande como para que los errores muestrales de la deriva génica sean desestimables, está lo suficientemente aislada como para que el efecto de las migraciones sea desestimable, no hay razones para pensar que el apareamiento no sea azaroso (por ejemplo no hay autopolinización) y el efecto de la mutación es desestimable (normalmente este es el caso puesto que las tasas de mutación son bajas). ¿Cómo explicamos que la frecuencia para esos alelos no es la esperada? Podemos apelar a TSN. Probablemente alguno de los alelos esté sufriendo una presión selectiva. Como veíamos, Darwin había propuesto TSN para dar una explicación histórica del origen de la adecuación. Hoy, TSN también tiene la función de aplicarse justamente en este caso. Uno de los alelos está teniendo más éxito que el otro en reproducirse porque incrementa la aptitud de los organismos que lo portan.

\section{TSN y GP, teorías distintas}

Si la presentación de HW dada en el apartado anterior es correcta, entonces queda claro que TSN y GP son teorías distintas. GP permite determinar uno de los conceptos de TSN, el de éxito reproductivo que, como veíamos, es un concepto TSN no teórico. GP funcionaría en la «base empírica» de TSN.

Sin embargo, como veíamos en la introducción del capítulo, textos de biología evolutiva, de historiografía de la biología y de filosofía de la biología, suelen oscilar a la hora de conceder un estatus independiente de GP a TSN. ¿Es posible considerar que GP brindó una versión cuantificada de lo que Darwin había presentado de manera cualitativa?

La siguiente analogía puede ser útil para discutir la cuestión. Del mismo modo que en un mundo newtoniano podría inferirse a partir de que una partícula se ha acelerado, que una fuerza - o una interacción de fuerzas - ha actuado, en un mundo en el que migración, deriva génica, mutación, apareamiento selectivo y selección natural fuesen las únicas fuerzas que actuaran sobre las frecuencias génicas de una población, uno podría inferir que la selección natural ha actuado a partir de que la frecuencia génica en una población no es la esperada y de la constatación de que ninguno de las otros factores tomados en cuenta en HW han intervenido. Pero señalar que hay razones para creer que la selección natural ha actuado no es equivalente a aplicar TSN, del mismo modo que en este 
supuesto mundo newtoniano, señalar que una fuerza o una combinación de fuerzas es la responsable del cambio de aceleración, no es equivalente a aplicar la mecánica clásica de partículas. Una aplicación de la mecánica clásica de partículas implica determinar el número y la naturaleza de las fuerzas en acción. De igual modo, aplicar TSN implica encontrar el rasgo que está siendo utilizado de manera más efectiva y la conexión entre tal rasgo y el éxito reproductivo, que yo he llamado «aptitud». Así como en el ejemplo newtoniano puede haber una combinación de fuerzas de diferente naturaleza actuando sobre una partícula, puede ocurrir lo mismo en la aplicación de TSN. La presión selectiva podría ser producida por una combinación entre, por ejemplo, supervivencia y capacidad de atracción de parejas de apareamiento. En conclusión, la mera determinación de la existencia y la fuerza de una presión selectiva no es equivalente a aplicar TSN.

Insisto, dada la estructura genética de una población, la mera predominancia de un rasgo fenotípico por sobre otro no permite inferir que los organismos que portan dicho rasgo están teniendo éxito en la competencia por dejar descendencia por sobre otros organismos con otros rasgos fenotípicos. Puede tratarse de un rasgo determinado por un alelo dominante, por ejemplo. Por lo tanto, lo que permite HW en GP es una sofisticación y mejora de la medición del éxito reproductivo diferencial.

\section{Vínculos interteóricos}

En el marco conceptual estructuralista se propone una clasificación de vínculos que dos teorías pueden tener (Balzer, Moulines, y Sneed 1987, cap. VI; Moulines 1991, cap. III.3). Me enfocaré en los vínculos interteóricos determinantes [presupposition links] (Balzer, Moulines, y Sneed 1987, pp. 57-62). La naturaleza de este tipo de vínculos es semántica. Son los vínculos por los cuales un concepto de una teoría es utilizado en otra, es decir, cuando en una teoría dada aparece un concepto que puede ser determinado utilizando otra teoría. Se llama a este tipo de vínculos «determinantes» para distinguirlos de los vínculos implicativos que son de naturaleza global, conectan leyes y no conceptos.

Concedamos lo máximo que se puede conceder a los que sostienen un lugar central de GP en la teoría evolutiva, esto es, que GP es necesaria para la determinación del éxito reproductivo de los individuos, y por lo tanto, necesariamente 
presupuesta en toda aplicación actual de $\mathrm{TSN}^{8}$. En este sentido habría un vínculo determinante que conecta el concepto de éxito reproductivo de TSN con el concepto de éxito reproductivo en GP — lo que suele llamarse en GP «éxito reproductivo», "presión selectiva» o "aptitud» [fitness] (que no habría que confundir con el concepto de aptitud de TSN).

\section{Conclusiones}

Existen varios trabajos que han señalado la independencia de estas dos teorías. Así, por ejemplo, Glymour sostiene:

La genética de poblaciones nos ha dado un conjunto de herramientas útiles para representar los cambios de frecuencia en las poblaciones naturales y para describir procesos de selección. Estos usos son importantes, incluso esenciales, en la mayor parte de la biología evolutiva y poblacional. Pero, mayormente por un accidente histórico, muchos biólogos y casi todos los filósofos han sobrestimado a la genética de poblaciones, en tanto, implícita o explícitamente, la genética de poblaciones es considerada como proveyendo el corazón formal de la maquinaria para describir procesos selectivos (Glymour 2006, p. 388).

Por su parte, Rosenberg afirma:

Los biólogos evolucionistas emplean la noción de «frecuencias génicas» como una unidad conveniente en la cual medir los resultados de varias fuerzas evolutivas... Dado que los genes proveen la unidad de medida del fenómeno al cual aplicamos la teoría de la selección natural, es inevitable que la genética de poblaciones sea central en la biología evolutiva. Pero por la mis-

8 Si este fuera el caso, tal vez habría que conceder que con la síntesis TSN sufrió un cambio esencial, puesto que los vínculos determinantes son esenciales a las teorías. En este sentido se podría decir que la teoría de la selección natural de Darwin y la actual son diferentes. Pero no afirmo que este sea el caso. Esto implicaría, por ejemplo, que el éxito reproductivo sólo puede ser determinado en casos de rasgos determinados genéticamente, y por genes en el sentido clásico. Lo cual podría implicar una restricción en el campo de aplicación de TSN. No podría aplicarse, por ejemplo, a rasgos de transmisión cultural. 
ma razón los termómetros son cruciales en la termodinámica: ellos reflejan efectos proximales que podemos medir, no causas últimas (Rosenberg 1994, pp. 110-111).

Ambos autores sostienen que GP, por sí misma, no es lo suficientemente explicativa en algún sentido. Considero que este trabajo permite reforzar estos puntos de vista desde una perspectiva de análisis de las teorías científicas. Efectivamente, GP no explica por qué un tipo de organismos está teniendo más éxito en la reproducción diferencial.

Vimos entonces, que GP y TSN son teorías distintas pero relacionadas. Así, TSN permite explicar por qué cierto tipo de organismos está teniendo más éxito en la reproducción diferencial que otros, hecho que puede ser determinado con la GP.

Si mi enfoque está en lo correcto, es posible afirmar entonces, por un lado, que ninguna de estas dos teorías (GP y TSN) es especialización de la otra. TSN ni se identifica ni se subsume ni reduce de ningún modo a GP. Nos encontramos frente a dos teorías distintas con vínculos entre sí, uno de los cuáles hemos examinado aquí. Por otro lado, contra los que pretendían, como veíamos, conceder un estatus privilegiado a GP en la teoría evolutiva, existe al menos una teoría independiente y distinta de GP en la teoría evolutiva: TSN. No podría, por lo tanto, considerarse a $\mathrm{H}-\mathrm{W}$ de ningún modo, la ley fundamental de la teoría evolutiva.

\section{Bibliografía}

Balzer, W., Moulines, C. U. y Sneed, J.D, 1987, An Architectonic for Science: the Structuralist Program. Dordrecht,/Lancaster, Reidel.

BARBADILLA, A., 1990, «La estructura de la teoría de la selección natural», en A. Ruiz, A. y M. Santos. (eds.), Temas Actuales de Biología Evolutiva, Barcelona: UAB.

BLANCO, D., 2008, «La naturaleza de las adaptaciones en la teología natural británica: análisis historiográfico y consecuencias metateóricas», Ludus Vitalis XVI (30), 2008, $3-26$.

DARWIN, C., 1872, The origin of species, 6th ed. London: John Murray.. 
Endler, J. A., 1986, Natural Selection in the Wild. Princeton, New Jersey: Princeton University Press.

FutuYMA, D. J., 1986, Evolutionary Biology - Second Edition. Sunderland, Massachusetts: Sinauer Associates, Inc.

GAYON, J., 1998, Darwinism's struggle for survival: heredity and the hypothesis of natural selection, Cambridge studies in philosophy and biology. Cambridge: Cambridge University Press.

GinNobili, S., 2006, La teoría de la selección natural darwiniana. Tesis de licenciatura. Filosofía, Buenos Aires: Universidad de Buenos Aires.

GinNOBILI, S., 2007a, «Darwinismo universal de dominio de aplicación restringido», en Lilian Al-Chueyr Pereira Martins, Maria Elice Brzezinski Prester, Waldir Stefano y R. de Andrade Martins (eds.), Filosofia e história da biologia 2, São Paulo: Fundo Mackenzie de Pesquisa (MackPesquisa), 427-443.

- 2007b, «Hay lo que queda. Sobre la presunta tautologicidad de la teoría de la selección natural.», Análisis Filosófico XXVII (1), 2007, 75-89.

- (por aparecer), «Adaptación y función», Ludus Vitalis XVII (31), 2009.

- (por aparecer), «Selección artificial, selección sexual, selección natural», Revista filosófica del sur I (1).

Ginnobili, S., y C. C. CARMAn, 2008, «Deferentes, epiciclos y adaptaciones», en, Filosofía e História da Ciência no Cone Sul. Seleção de trabalhos do $5^{\circ}$ Encontro, Campinas: Associação de Filosofia e História da Ciência do Cone Sul (AFHIC), 2008.

GLYMOuR, B. 2006, «Wayward Modeling: Population Genetics and Natural Selection», Philosophy of Science 73 (4), 2006:369-389.

Gould, S. J., y E. S. VRBA, 1982, «Exaptation-A Missing Term in the Science of Form», Paleobiology 8 (1), 1982:4-15.

LORENZANO, P., 2005, «Ejemplares, modelos y principios en la genética clásica», Scientiae Studia 3 (2), 2005:185-203.

- 2008, «Bas C. van Fraassen y la ley de Hardy-Weinberg: una discusion y desarrollo de su diagnóstico.», Principia. Revista Internacional de Epistemologia 12 (2), 2008, 121-154.

LloYD, E. A. 1994, The Structure and Confirmation of Evolutionary Theory. New Jersey: Princeton University Press.

Moulines, U. 1991, Pluralidad y recursión. Madrid: Alianza Universidad. 
Rosenberg, A. 1994, Instrumental Biology or The Disunity of Science. Chicago and London: The University of Chicago Press.

Ruse, M. 1973, The Philosophy of Biology. London: Hutchinson \& Co.

Thompson, P. 1989, The Structure of Biological Theories. New York: State University of New York Press.

Recibido: 8/06/2009

Revisado: 19/06/2009 
